Disponível em

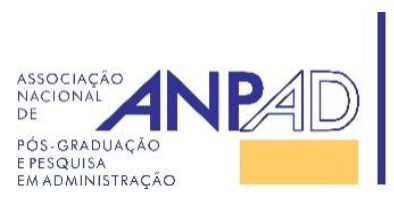

http://www.anpad.org.br/rac

RAC, Rio de Janeiro, v. 21, n. 6, art. 4, pp. 811-831, Novembro/Dezembro, 2017 http://dx.doi.org/10.1590/1982-7849rac2017160319

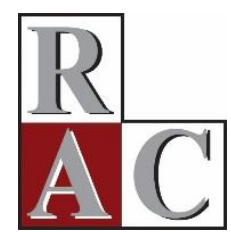

\title{
Formação, Disseminação e Colaboração: Internacionalização em Administração de Sistemas de Informação
}

\author{
Academic Education, Research Dissemination and Collaboration: Internationalization \\ in Management Information Systems
}

Eduardo H. Diniz ${ }^{1}$ José Eduardo Ricciardi Favaretto ${ }^{1}$ Henrique Pontes Gonçalves de Oliveira ${ }^{1}$ Debora Richter Brólio ${ }^{1}$

Fundação Getulio Vargas ${ }^{1}$

Artigo recebido em 09.11.2016. Última versão recebida em 13.03.2017. Aprovado em 22.03.2017. Publicado online em 11.10.2017. 


\title{
Resumo
}

Este artigo revela o perfil de internacionalização dos pesquisadores brasileiros no campo de Administração de Sistemas de Informação (ADI) com base em análise de três dimensões: Formação, Disseminação de pesquisa e Colaboração em redes internacionais. Baseado na coleta de dados provenientes da Plataforma Lattes, Google Scholar e dos websites das instituições de ensino, referente a um total de 554 pesquisadores com doutorado completo, um grupo de 50 pesquisadores brasileiros neste campo foi identificado com algum grau de inserção internacional. Este estudo revela que os pesquisadores mais internacionalizados do campo são majoritariamente provenientes de instituições das regiões Sudeste (66\%) e do Sul (28\%) do País. Apenas uma parcela (16\%) desses pesquisadores realizou doutorado no exterior, embora outros tenham tido experiências internacionais por intermédio de pós-doutorado (38\%), cursos de extensão (45\%) ou bolsa sanduíche (18\%). A Disseminação internacional dos trabalhos deste grupo de pesquisadores cresceu 65\% no período entre 2010 e 2015 , com publicação em periódicos e participação em congressos internacionais. A rede de Colaboração internacional deste grupo de pesquisadores também foi identificada na pesquisa.

Palavras-chave: internacionalização; sistemas de informação; publicação; formação acadêmica; colaboração acadêmica.

\begin{abstract}
This research evaluates the internationalization profile of Brazilian researchers in the Management Information Systems field based on analysis of three dimensions: academic education, research dissemination and collaboration in international networks. Based on data collection from the Brazilian Lattes Platform (an online database of curriculum vitae), Google Scholar and the websites of education institutions, regarding a total of 554 researchers with doctorate degrees, a group of 50 Brazilian researchers with some level of international integration was identified. This study reveals that the most internationalized researchers in the field are mostly from institutions in the Southeast $(66 \%)$ and South $(28 \%)$ of Brazil. Only a few $(16 \%)$ of these researchers did their doctorate abroad, although others had international experience through postdoctoral studies (38\%), extension courses (45\%) or sandwich scholarships (18\%). The international dissemination of the work of this group of researchers grew $65 \%$ in the period between 2010 and 2015, with publication in periodicals and participation in international conferences. This group's international collaboration network was also identified in the research.
\end{abstract}

Key words: internationalization; information systems; publication; academic education; academic collaboration. 


\section{Introdução}

Na grande área de conhecimento da Administração, o campo de estudo acadêmico identificado como Management Information Systems (MIS) - resumidamente, Information Systems (IS) ou Sistemas de Informação (SI), estuda o impacto da Tecnologia de Informação e Comunicação (TIC) nas organizações e a influência de seus artefatos nas interações com indivíduos, grupos e sociedade (Orlikowski \& Iacono, 2001). Neste artigo esses pesquisadores serão identificados como sendo do campo de Administração de Sistemas de Informação (ADI), nome de uma das divisões acadêmicas do EnANPAD, principal congresso da área de Administração no Brasil.

Se a pesquisa acadêmica brasileira no campo de ADI na forma como ela é produzida e comunicada já atingiu um patamar relativamente estruturado no âmbito nacional, com uma produção regular e alguns Programas de Pós-Graduação (PPGs) com notas 7 e 6 na Coordenação de Aperfeiçoamento de Pessoal de Nível Superior (CAPES, 2016a), a inserção internacional dos pesquisadores desses programas ainda está em fase embrionária e precisa ser melhor estudada. Embora se saiba que entre os principais programas do campo de ADI no Brasil militam pesquisadores com grau de articulação relativamente estável com alguns centros internacionais de produção no campo, não se conhece nenhum estudo que mapeie as características e os impactos desta internacionalização no País em termos temáticos e teóricos, nem do potencial de contribuição da produção nacional deste campo em âmbito internacional.

Investigar a inserção internacional em ADI é também importante pela conhecida relação entre colaboração internacional e a relevância da produção científica (Katz \& Martin, 1997), pois o esforço para publicação internacional pode ser menos relevante se não estiver associado a estratégias de pesquisa baseadas em colaboração internacional (Meneghini, Packer, \& Nassi-Calo, 2008). Assim, se quisermos aumentar a relevância, qualidade e reputação da pesquisa em ADI no Brasil, é preciso investigar as estratégias de desenvolvimento das relações de colaboração internacional da área, inclusive porque o investimento para promover o trabalho colaborativo incorre em altos custos que devem ser gerenciados com parcimônia e objetividade. Essa discussão torna-se ainda mais relevante para o Brasil, país com tradição acadêmica limitada e dificuldades para sustentar financiamento científico.

Para realizar a investigação sobre o perfil da internacionalização do campo ADI, este estudo foi baseado na análise de três dimensões que representam as oportunidades de articulação internacional de um pesquisador (Diniz, Favaretto, Oliveira, \& Brólio, 2016). A primeira dimensão é a sua Formação acadêmica, a partir da identificação do país no qual o pesquisador concluiu o seu doutorado (ou PhD), mestrado, participou de programas de post-doc, bolsa sanduíche ou algum curso de extensão. A segunda dimensão é a capacidade de Disseminação internacional dos trabalhos de pesquisa a partir do mapeamento de artigos que foram publicados e/ou apresentados em periódicos e congressos internacionais. A terceira dimensão é a Colaboração, identificada pela participação do pesquisador em grupos ou projetos de pesquisa financiados internacionalmente ou que contam com a participação de pesquisadores de outros países, sua atuação como avaliador ou editor associado em periódicos e congressos internacionais e, ainda, a participação em associações científicas internacionais. Essas três dimensões podem ser ilustradas pelo diagrama conceitual da Figura 1. 


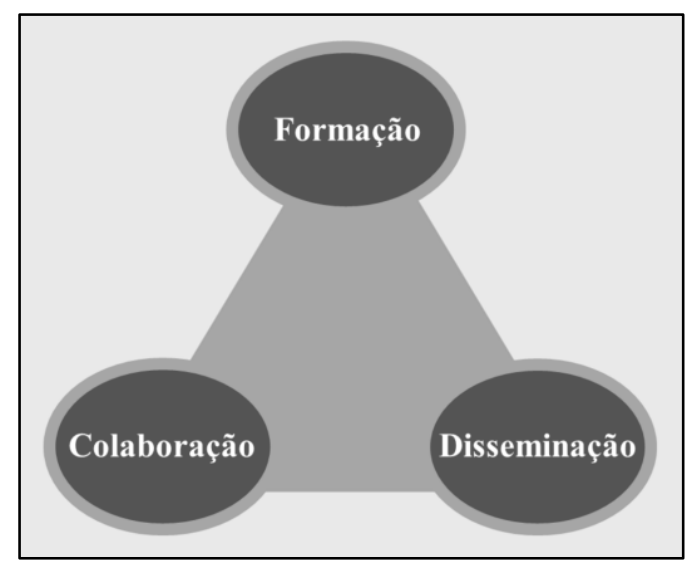

Figura 1. Diagrama Conceitual da Pesquisa com suas Três Dimensões: Formação, Disseminação e Colaboração Internacional

Fonte: Elaborada pelos autores.

A Figura 1 representa o diagrama utilizado para o embasamento teórico e conceitual estudado nesta pesquisa, nas três dimensões de análise - Formação, Disseminação e Colaboração internacional, o qual guiou a realização de suas etapas posteriores e buscou responder à seguinte questão de pesquisa: Qual o perfil de atuação internacional dos pesquisadores brasileiros em função das três dimensões consideradas (Formação, Disseminação de pesquisa e Colaboração internacional)?

É importante ressaltar que o perfil de atuação traçado nesta pesquisa foi baseado principalmente na coleta das informações da Plataforma Lattes de cada pesquisador, plataforma considerada internacionalmente como um exemplo de boas práticas no fornecimento de dados de alta qualidade sobre pesquisadores e instituições acadêmicas brasileiras (Lane, 2010) e comumente utilizada como fonte de dados secundários na redação de artigos científicos (Dias, Moita, \& Dias, 2016; Perlin, Santos, Imasato, Borenstein, \& Silva, 2017). Embora seja reconhecido que outros fatores possam também influenciar no processo de internacionalização, como políticas de incentivo da instituição à qual o pesquisador está vinculado, ações desenvolvidas por organismos de fomento e mesmo outros aspectos contextuais e regionais, estes elementos não foram considerados nesta pesquisa. Assim, o foco neste artigo foi o mapeamento das ações individuais já realizadas pelos pesquisadores (entre 2010 e 2015), sem considerar outros fatores.

Este artigo apresenta na próxima seção uma revisão de literatura que contextualiza o campo de ADI e as três dimensões de análises utilizadas neste estudo. A seguir, a metodologia de coleta e as técnicas de análise de dados são detalhadas para, na sequência, se apresentar os resultados obtidos desta coleta. Na seção seguinte discutem-se os resultados, e o artigo se encerra com alguns comentários e indicações de continuidade do estudo.

\section{Revisão de Literatura}

O campo acadêmico de Administração de Sistemas de Informação tem origem na década de 60 , oriundo da interdisciplinaridade de diferentes áreas de estudo, tais como ciência da computação, operações, contabilidade e estudos organizacionais/administração (Benbasat \& Zmud, 2003; Bryant, Black, Land, \& Porra, 2013; Hirschheim \& Klein, 2012; Zhang, 2015), e da necessidade de atender às atividades práticas nas organizações, ao abastecê-las com novos conhecimentos para utilização da Tecnologia de Informação (TI) em suas estruturas (Gannon, 2013; King \& Lyytinen, 2006).

Um recente resgate histórico das pesquisas acadêmicas brasileiras deste campo, nas temáticas identificadas nos artigos publicados nos anais do EnADI e do EnANPAD - divisão de Administração da Informação (ADI) no período de 1994 a 2013 -, trouxe para discussão que esse campo no Brasil passou 
por dois grandes períodos de tempo, bem distintos: de 1994 a 2002, considerado de alavancagem da área no País, e de 2003 a 2013, considerado como de formação e consolidação de grupos de pesquisa (Freitas, Becker, Martens, \& Marcolin, 2014). Mas apesar dos aspectos identificados neste estudo, e mesmo em outros anteriores realizados também no Brasil (Graeml \& Macadar, 2010; Graeml, Macadar, Guarido, \& Rossoni, 2010; Hoppen \& Meirelles, 2005; Macadar \& Graeml, 2010; Rodrigues \& Ludmer, 2005; Rossoni \& Hocayen-da-Silva, 2009), não foram localizados na literatura acadêmica artigos que relatassem a respeito do processo de internacionalização de pesquisadores brasileiros de ADI, demonstrando ser uma temática oportuna para investigação.

Ao longo desses mais de 50 anos, a discussão sobre a identidade do campo periodicamente reaparece como tema de debate entre seus pesquisadores (Benbasat \& Zmud, 2003; Hirschheim, Saunders, \& Straub, 2012; Ives, Valacich, Watson, \& Zmud, 2002; Sidorova, Evangelopoulos, Valacich, \& Ramakrishnan, 2008; Wade, Biehl, \& Kim, 2006). Há questões sobre a carência de um extenso e robusto corpo teórico nativo (Straub, 2012; Weber, 2012; Zmud, 1998) pelo possível excesso de aproveitamento de teorias oriundas de outras áreas / disciplinas (Bernroider, Pilkington, \& Córdoba, 2013; Clarke, 2008; Grover \& Lyytinen, 2015). Há também certa dificuldade em se delimitar consensualmente o corpo de conhecimento e habilidades que deveriam ser consideradas centrais em estudos de SI (King \& Lyytinen, 2006).

A internacionalização do campo de SI é também vista como um sinal de maturidade desejável. Saunders (2006), em editorial do MIS Quartely sobre a necessidade de expansão do perfil global deste periódico, reconhece que há um viés no campo e, para eliminá-lo, aponta a necessidade de internacionalização de temáticas e de revisores, "abertos a diferentes perspectivas e metodologias" (p. v). A autora ressalta também a necessidade de maior participação dos membros da comunidade internacional de Sistemas de Informação nesse processo de internacionalização, necessária para manter este campo acadêmico sintonizado com o alto grau de internacionalização existente no ambiente de negócios.

De fato, o viés apontado por Saunders no principal periódico da área também se reflete na Association for Information Systems (AIS) - associação profissional e científica que agrega cerca de metade da população estimada de milhares de acadêmicos de escolas de negócios especializados em Sistemas de Informação. Dos associados da AIS, quase 50\% atuam em escolas localizadas na América do Norte, e apenas cerca de 30 associados são pesquisadores brasileiros (AIS, n.d.a).

Para buscar maior visibilidade no cenário internacional, segundo Heinzl, Winter e Bichler (2015), há basicamente três formas primárias de determinada comunidade local de pesquisa exportar conhecimento: "publicar a pesquisa em periódicos ou congressos internacionais do campo (os chamados international outlets), participar na condução de projetos internacionais de pesquisa ou fazer a própria transferência física do acadêmico para o exterior" (p. 226). Assim, a partir da contextualização da identidade, do impacto e da internacionalização na pesquisa do campo de estudos de SI, adicionada da mobilidade internacional de pesquisadores e de suas pesquisas (Chua, Cao, Cousins, \& Straub, 2002; Clark, Au, Walz, \& Warren, 2011; Dennis, Valacich, Fuller, \& Schneider, 2006), pode-se entender a internacionalização do campo de SI a partir de três dimensões: experiência internacional ao longo da Formação dos pesquisadores, avaliação da Disseminação internacional da sua produção científica e envolvimento dos pesquisadores da comunidade em redes internacionais de Colaboração. A seguir, um aprofundamento dessas três dimensões de análise.

\section{Formação internacional}

A internacionalização vista como um procedimento bidirecional oferece um enorme potencial para uma comunidade local estender o impacto do conhecimento produzido para além de seu próprio limite geográfico (Heinzl, Winter, \& Bichler, 2015), permitindo também importar e aproveitar novas ideias de outras partes do mundo, para serem utilizadas na pesquisa e no ensino local. Apesar do benefício da internacionalização e da participação de pesquisadores de outras partes do mundo no mainstream do campo de estudos de SI, não se pode correr o risco de deixar perder ou de não se 
aproveitar as referências locais ou tradições do país de origem (Heinzl et al., 2015; Pozzebon, Diniz, \& Reinhard, 2011).

Um estudo bibliométrico com dados coletados de um conjunto de 11 principais periódicos internacionais do campo de SI, entre os anos de 2005 e 2009, investigou pesquisadores líderes do campo com 5 ou mais artigos publicados (90\% já com título de doutor ou em fase de titulação), as universidades a que estes estavam vinculados, bem como as escolas onde eles obtiveram/pleiteavam seus títulos de doutor (Clark et al., 2011). Este estudo constatou a liderança de determinadas escolas onde os pesquisadores receberam seus títulos de doutores, além das vinculações atuais dos autores. Dentre as escolas líderes que foram explicitamente mencionadas no estudo estão a Georgia State University, a City University of Hong Kong, a University of Houston, a University of Minnesota, a University of Arizona e a Purdue University. Isso reitera que um dos componentes de sucesso na publicação de artigos nos periódicos de ponta do campo internacional de SI é a influência na formação de doutoramento do pesquisador ter ocorrida em escolas nos EUA, tradicionalmente voltadas ao ensino e pesquisa no campo de Sistemas de Informação.

Para além das universidades mencionadas, majoritariamente localizadas nos EUA, Mumford (2006) apresenta o histórico de um conjunto de princípios humanísticos que contribuíram significativamente para o desenvolvimento do campo de SI, ampliando a disseminação de métodos e abordagens interpretativistas. A autora revela, em seu artigo, como essa nova visão de pesquisa foi sendo abordada inicialmente por pesquisadores atuantes em escolas localizadas na Noruega, Suécia e Dinamarca, para gradativamente ser adotada em outros países da Europa e nos EUA, e de lá para os outros continentes.

Fica claro, pelo que foi dito acima, que há uma grande influência de algumas poucas escolas e países nos trabalhos publicados no campo de SI. É de se esperar que alunos formados nessas escolas e países tenham tido a oportunidade de se integrar melhor à comunidade internacional que atua mais próximo aos focos de maior reverberação do conhecimento na área. Por outro lado, a aproximação física de pesquisadores brasileiros com os desses países permite potencializar a visibilidade da pesquisa realizada no país tendo foco no cenário internacional.

\section{Disseminação internacional da produção científica}

Quando rankings de publicações e contagens de citações parecem dominar a avaliação da pesquisa (Bichler, Heinzl, \& Winter, 2015), a publicação em outlets internacionais ganha muita importância. Como o campo de SI é essencialmente multidisciplinar, seja pelas teorias adotadas, pelos métodos aplicados ou pelos variados temas abordados, há uma diversidade intrínseca no conhecimento produzido (Benbasat \& Zmud, 2003), e os pesquisadores do campo enfrentam desafios adicionais para publicar internacionalmente. Como pesquisadores de outras áreas, além de serem capazes de convencer seus pares internacionais, muitas vezes com visões muito divergentes, devem também estar dispostos a publicar fora de sua comunidade de origem, dado o hibridismo de temáticas estudadas (Heinzl et al., 2015).

Com o objetivo de definir mais claramente qual deveria ser a lista de periódicos de referência para a área e que deveriam ser considerados em casos de contratação e de promoção de pesquisadores, um College of Senior Scholars foi nomeado dentro da AIS (n.d.b). Estes pesquisadores, reconhecidos pela sua liderança na área, definiram uma senior basket contendo os Top-8 principais periódicos internacionais do campo de SI. Algumas variações desta lista aparecem na literatura, em alguns casos reduzindo a lista para 6 ou 3 (Lin \& Gregor, 2009) e, em outros, ampliando para 11 (Clark et al., 2011) ou até 13 periódicos (Chan, Guness, \& Kim, 2015).

Apesar da orientação mais ou menos limitada desta lista de periódicos, o que também regula o grau de dificuldade de pesquisadores de fora dos EUA de publicar nos top-journals da área, a internacionalização da pesquisa em SI vem ocorrendo de maneira gradativa, em periódicos que vão além daqueles recomendados pela AIS (Chan et al., 2015). Mas, ainda assim, o domínio intenso das universidades e pesquisadores norte-americanos também é destaque nestes outros periódicos.

RAC, Rio de Janeiro, v. 21, n. 6, art. 4, pp. 811-831, Novembro/Dezembro, 2017, www.anpad.org.br/rac (cc) EY 
Foi também identificado um contraponto na percepção dessas classificações (ranking) de periódicos, com a finalidade de submissão de artigos para publicações. Para pesquisadores da London School of Economics (LSE), publicar em periódicos mainstream da área nem sempre é apropriado, pois há outros em que o impacto (acadêmico e prático) das pesquisas podem ser maiores (Willcocks, Whitley, \& Avgerou, 2008).

Com os congressos a situação não é muito diferente dos periódicos. Há uma lista de três grandes congressos promovidos pela AIS: ICIS, AMCIS e PACIS. Além desses, quatro capítulos da AIS organizam também congressos apoiados por essa associação, entre eles, e pela primeira vez, a partir da instalação do Capítulo Brasileiro da AIS no Brasil em 2016 (Pritchett, 2016), está incluído o BRAIS, identificado localmente como CONTECSI, International Conference on Information Systems and Technology Management, realizado na cidade de São Paulo. Outros 10 congressos promovidos por organizações que são afiliadas à AIS também estão na lista de eventos que devem ser considerados prioritários para o campo.

Além dos congressos com alguma ligação com a AIS, a International Federation for Information Processing, IFIP, embora mais ligada à área de Ciência da Computação, possui entre seus 14 comitês técnicos alguns grupos de trabalho (working groups, ou simplesmente WG) que cobrem temas comumente tratados na área de SI, como o WG 8.5 (Information Systems in Public Administration), o WG 8.2 (The Interaction of Information Systems and the Organization) e o WG 9.4 (Social Implications of Computers in Developing Countries), cada um deles com seus próprios congressos. Não se pode deixar de mencionar ainda o Hawaii International Conference on System Sciences (HICSS), o mais antigo congresso da área, com a sua $50^{\mathrm{a}}$ edição promovida em janeiro de 2017. Além desses, é claro, existem congressos mais generalistas da área de Administração e que possuem trilhas que concentram os trabalhos relacionados ao campo de SI, dos quais o mais importante é o Academy of Management Annual Meeting (AOM).

\section{Colaboração internacional em redes de pesquisadores}

A coautoria em um artigo é considerada a manifestação mais comum de colaboração intelectual no meio acadêmico, apesar da existência de muitas outras formas de colaboração, como: a revisão de artigos em periódicos, a organização de um congresso, a edição de um periódico, a participação em grupos de pesquisa e a participação em projetos de pesquisas internacionais. Uma rede de coautoria nos proporciona uma janela para o desenvolvimento do capital social na comunidade de SI (Xu, Chau, \& Tan, 2014). É reiterado por Heinzl et al. (2015) que a condução da pesquisa com colegas internacionais quase sempre só é viável quando subsidiada por fundos financeiros provenientes de agências de fomento à pesquisa, até mesmo de maneira bilateral.

Enquanto a comunidade de SI vem crescendo internacionalmente, é razoável esperar que a sua localização, em diferentes regiões do mundo, traga particularidades definidas localmente, tais como apelos de pesquisa, interesses e orientações para a publicação dos trabalhos (Stein, Galliers, \& Whitley, 2016). Em estudo baseado na análise de citações de artigos de estudiosos alemães da área do conhecimento da Administração (Eisend \& Schmidt, 2014), foi comprovado que o sucesso na estratégia de internacionalização usada por estes acadêmicos ocorria pela colaboração internacional e pela disponibilidade dos chamados recursos do conhecimento (domínio da língua estrangeira, experiência em pesquisa e conhecimento do mercado estrangeiro).

Em outro estudo que avaliou taxas de citação em periódicos de SI durante 7 anos, de uma forma geral foi percebido que a coautoria passou a ser incrementada ao longo dos anos, e a quantidade de coautores estava relacionada à influência acadêmica dos autores (Gallivan \& Ahuja, 2015). Foi notado que, além do domínio predominante dos pesquisadores norte-americanos na publicação científica nos periódicos Top-3 do campo de IS, os artigos em coautoria estão em ascensão nesses três principais periódicos (Clark et al., 2011), reiterando que a participação em redes de colaboração pode alavancar o sucesso na inserção de pesquisas neste grupo de periódicos de ponta. 


\section{Metodologia e Coleta de Dados}

Para avaliar a inserção internacional dos pesquisadores brasileiros no campo de estudos de SI, a metodologia adotada para estruturar a coleta e o refinamento de dados dos pesquisadores estudados foi dividida em duas etapas: na primeira etapa foram identificados os pesquisadores com atuação no campo de estudos e, na segunda, foram utilizados filtros de exclusão para selecionar, dentre os identificados, aqueles que possuíam atuação internacional mais destacadas, com base nas dimensões teóricas da pesquisa - Formação internacional, Disseminação da produção científica e Colaboração em redes de pesquisa internacionais.

\section{Primeira etapa da coleta de dados - Lista de 554 pesquisadores}

Nesta primeira etapa da coleta de dados foram identificados os pesquisadores brasileiros com formação mínima em doutorado pela adoção de quatro critérios de seleção, conforme informados na Tabela 1.

Tabela 1

Critérios Adotados para a Identificação e Seleção dos Pesquisadores Doutores do Campo de ADI (Primeira Etapa)

\begin{tabular}{|c|c|c|}
\hline Critério & Descrição & Fonte \\
\hline $\begin{array}{l}\text { 1.PPGs com Linha de } \\
\text { Pesquisa em ADI }\end{array}$ & $\begin{array}{l}\text { Pesquisadores que atuam em instituições } \\
\text { que mantêm Programas de Pós- } \\
\text { Graduação (PPGs) de doutorado na área } \\
\text { de Administração de Empresas, com } \\
\text { linha de pesquisa em ADI, e nota igual } \\
\text { ou superior a 3, atribuída pela CAPES }\end{array}$ & $\begin{array}{l}\text { Coordenação de Aperfeiçoamento de } \\
\text { Pessoal de Nível Superior (CAPES, } \\
\text { 2016a) e websites das Instituições de } \\
\text { Ensino Superior (IES) - páginas com a } \\
\text { identificação de seus pesquisadores }\end{array}$ \\
\hline $\begin{array}{l}\text { 2. Coordenação ou } \\
\text { Liderança de trilha na } \\
\text { área temática de ADI }\end{array}$ & $\begin{array}{l}\text { Pesquisadores que atuam em PPGs de } \\
\text { doutorado na área de Administração de } \\
\text { Empresas e tiveram a função de } \\
\text { coordenador ou líder de trilha na área } \\
\text { temática de ADI nos congressos do } \\
\text { EnANPAD ou EnADI, no período de } \\
2010 \text { a } 2015 \text {, não considerados no } \\
\text { critério } 1\end{array}$ & $\begin{array}{l}\text { Associação Nacional de Pós-Graduação e } \\
\text { Pesquisa em Administração (ANPAD, } \\
\text { n.d.) }\end{array}$ \\
\hline $\begin{array}{l}\text { 3.Publicação no } \\
\text { EnANPAD ou EnADI }\end{array}$ & $\begin{array}{l}\text { Pesquisadores que publicaram artigos } \\
\text { completos nos congressos do EnANPAD } \\
\text { ou no EnADI, no período de } 2010 \text { a } \\
2015 \text {, não considerados nos critérios } 1 \text { e } 2\end{array}$ & $\begin{array}{l}\text { Associação Nacional de Pós-Graduação e } \\
\text { Pesquisa em Administração (ANPAD, } \\
\text { n.d.) }\end{array}$ \\
\hline $\begin{array}{l}\text { 4. Publicação nos } \\
\text { periódicos JISTEM ou } \\
\text { RESI }\end{array}$ & $\begin{array}{l}\text { Pesquisadores (com título de doutor) que } \\
\text { publicaram artigos nos periódicos } \\
\text { JISTEM ou RESI no período de } 2010 \text { a } \\
2015 \text {, não considerados nos critérios } 1,2 \\
\text { e } 3\end{array}$ & $\begin{array}{l}\text { Journal of Information Systems and } \\
\text { Technology Management (JISTEM, n.d.) } \\
\text { e } \\
\text { Revista Eletrônica de Sistemas de } \\
\text { Informação (RESI, 2017) }\end{array}$ \\
\hline
\end{tabular}

Nota. Fonte: Elaborada pelos autores.

A Tabela 1 informa os critérios utilizados para a identificação e seleção dos pesquisadores doutores do campo de ADI, durante a primeira etapa da coleta de dados desta pesquisa. Pelo critério 1, foram identificados pesquisadores com atuação em Instituições de Ensino Superior (IES) que mantenham PPG de doutorado em Administração de Empresas e que declaradamente participam de linha de pesquisa ativa em ADI nestas instituições. O critério 2 permitiu identificar pesquisadores 
atuantes em IES com PPG de doutorado em Administração de Empresas e que possuíam participação na coordenação ou liderança de trilha da área temática de ADI nos congressos do EnANPAD e/ou EnADI, no período de 2010 a 2015. Já o critério 3 identificou pesquisadores que possuíam publicação de artigo científico nos congressos do EnANPAD e/ou no EnADI, no período de 2010 a 2015. Por fim, com a utilização do critério 4, foi possível identificar pesquisadores que publicaram artigos nos periódicos brasileiros Journal of Information Systems and Technology Management (JISTEM) ou Revista de Sistema de Informação (RESI), no período de 2010 a 2015.

A partir das IES listadas pela CAPES (2016a) e pela visita às páginas de seus websites contendo a identificação de seus pesquisadores (critério 1), foi possível gerar uma lista de nomes. Com os critérios 2, 3 e 4 também adotados, pela visita aos respectivos websites das demais fontes da pesquisa (ANPAD, n.d.; JISTEM, n.d.; RESI, 2017), essa lista foi ampliada para um número total de 554 pesquisadores. Assim, para confirmar todas as informações destes pesquisadores, foi utilizada a Plataforma Lattes (Conselho Nacional de Desenvolvimento Científico e Tecnológico [CNPq], n.d.), onde também foram coletados os respectivos dados de formação e produção científica de todos eles.

\section{Segunda etapa da coleta de dados - Lista de 50 pesquisadores}

Na segunda etapa da coleta de dados, com o objetivo de selecionar na lista dos 554 pesquisadores - obtida na primeira etapa da coleta de dados - aqueles que tinham participação internacional mais destacada e envolvimento declarado com o campo de pesquisa de Sistemas de Informação, foram aplicados três filtros de exclusão - ilustrados pela Figura 2 - na seguinte ordem: Filtro 1 - pesquisadores que não possuíam formação internacional ou produção internacional declarada em seu currículo Lattes (CNPq, n.d.); Filtro 2 - pesquisadores que apresentaram produção científica total menor que 5 publicações, entre 2010 e 2015, em congressos internacionais ou periódicos internacionais (CAPES, 2017; Journal Citations Report [JCR], 2017; SCImago Journal Rank [Scimago Journal \& Country Rank - SJR], 2017); Filtro 3 - pesquisadores que declaram explicitamente ser de linhas de pesquisa fora da Área de Administração e do campo de SI, confirmado pelo Google Scholar Search Authors (Google Scholar, n.d.), currículo Lattes (CNPq, n.d.) ou pelas páginas dos websites das IES onde eles atuam.

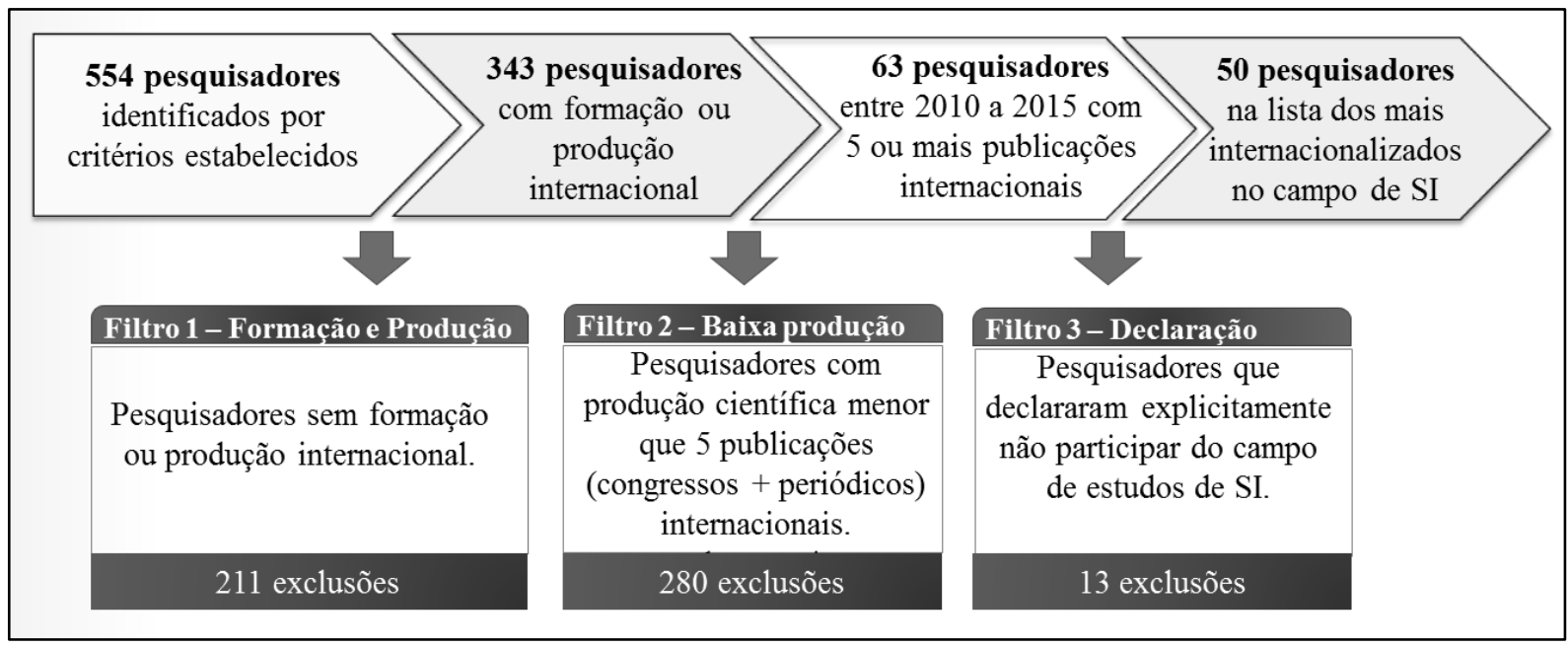

Figura 2. Fluxo dos Critérios e Filtros Aplicados para Resultar em uma Lista de 50 Pesquisadores com mais Projeção Internacional - Segunda Etapa da Coleta de Dados Fonte: Elaborada pelos autores.

A Figura 2 ilustra o fluxo dos critérios adotados na aplicação de três filtros de exclusão, partindo de uma lista inicial de 554 pesquisadores, com doutorado completo, que atenderam aos critérios estabelecidos na primeira etapa da coleta de dados. Após a aplicação do primeiro filtro, que selecionou apenas os pesquisadores com formação ou produção acadêmica internacional, chegou-se a um número de 343 pesquisadores. Com a aplicação do segundo filtro (já nesta lista de 343 pesquisadores), foram excluídos os pesquisadores com menos de 5 publicações internacionais (somados periódicos e 
congressos) - no período de 2010 a 2015, resultando em uma nova lista, agora com 63 pesquisadores. Nesta última lista foi aplicado o terceiro filtro, para excluir os pesquisadores que não declararam explicitamente a sua atuação no campo de estudos de SI - por exemplo, foram excluídos os que declararam pertencer aos campos de Ciência da Computação, Engenharia da Produção, Engenharia de Software ou Direito, ou seja, os que têm vínculos de pesquisa não pertencentes aos da área de Administração e campo de MIS (Hirschheim \& Klein, 2012; Zhang, 2015).

Como resultado da aplicação dos três filtros de exclusão, chegou-se a uma lista de 50 pesquisadores sobre os quais foi realizada uma investigação mais detalhada referente à sua inserção internacional.

Outro aspecto também considerado através de critérios de seleção foi o cômputo da totalização da produção científica avaliada nesta pesquisa. Os periódicos onde os artigos dos pesquisadores foram publicados deveriam estar listados ao menos em uma das três plataformas de classificação de periódicos: Sucupira Qualis (CAPES, 2016b), Scopus - SCImago (SJR, 2017) ou Thomson Reuters (JCR, 2017) como detalhado na Tabela 2.

Tabela 2

Plataformas de Classificação e Critérios Adotados para Aceitação dos Periódicos nas Totalizações deste Estudo

\begin{tabular}{|c|c|c|}
\hline Plataforma de Classificação & Critério de Aceitação & Fonte \\
\hline 1. Sucupira Qualis & $\begin{array}{l}\text { Periódicos com classificação no Qualis } \\
\text { 2014, igual ou superior a B3 }\end{array}$ & $\begin{array}{l}\text { Documento de avaliação da área de } \\
\text { Administração - (CAPES, 2017) e } \\
\text { Plataforma Sucupira - Periódicos } \\
\text { Qualis (CAPES, 2016b) }\end{array}$ \\
\hline 2. Scimago SJR & $\begin{array}{l}\text { Periódicos não classificados no Qualis } \\
\text { 2014, mas classificado no SJR } 2015\end{array}$ & $\begin{array}{l}\text { Scimago Journal \& Country Rank - } \\
\text { Scopus (SJR, 2017) }\end{array}$ \\
\hline 3. Impact Factor JCR & $\begin{array}{l}\text { Periódicos não classificados no Qualis } \\
\text { 2014, mas classificados no JCR } 2015\end{array}$ & $\begin{array}{l}\text { Fator de Impacto - Journal Citations } \\
\text { Report Thomson Reuters (JCR, 2017) }\end{array}$ \\
\hline
\end{tabular}

Nota. Fonte: Elaborada pelos autores.

A Tabela 2 resume os nomes das plataformas de classificação de periódicos, os critérios adotados e as fontes consultadas para a validação e aceitação dos periódicos nas totalizações das publicações de artigos deste estudo. Para os periódicos serem identificados como pertencentes à grande área de Administração, o comitê de avaliação desta área na CAPES (2017) usou na definição do extrato Qualis A1 de 2014 (CAPES, 2016b), além de ponderações numéricas das bases Thomson Reuters (JCR >1,4) ou Scopus Scimago (H-Scopus >24) - aquela que fosse a mais favorável - também a classificação das categorias do periódico em cada uma dessas bases. Ou seja, o periódico deveria estar obrigatoriamente classificado na base da Thomson Reuters (como pertencente às categorias: Business; Business Finance; Management; Hospitality, Leisure, Sports \& Tourism; Public Administration), ou na base Scopus' Subject area (como sendo: Business, Management \& Accounting; Decision Sciences; Economics, Econometrics and Finance; Social Sciences), caso contrário, receberia a classificação Qualis A2. O mesmo artifício foi adotado para a definição do extrato Qualis A2, com o valor do JCR do periódico entre 0,7 e 1,4 ou H-Scopus entre 9 e 24 (considerado o mais favorável), além também de o periódico ser classificado nestas bases nas categorias aceitas como Administração, caso contrário ficaria classificado no estrato B1.

Com relação aos congressos, foram considerados apenas trabalhos apresentados em congressos internacionais do campo de estudos de Sistemas de Informações ou aqueles da área com alguma trilha específica de SI, cuja edição tenha sido realizada fora do Brasil. 


\section{Apresentação dos Resultados}

Esta pesquisa identificou ao todo 20 instituições (Tabela 3) que possuíam programas de mestrado e doutorado na área de Administração de Empresas - com nota maior ou igual a 3 pela CAPES (2016a). Dentre estas instituições, três foram identificadas como possuidoras de linha de pesquisa em Administração e Tecnologia de Informação - são elas: FGV-SP, USP-SP e Universidade Federal do Rio Grande do Sul. Com a utilização dos demais critérios de seleção (Coordenação ou Liderança de trilha de ADI; Publicação no EnANPAD/EnADI e Publicação no JISTEM/RESI - descritos na Tabela 1), pesquisadores com atuação no campo de estudos de SI foram localizados em outras dezessete instituições.

Tabela 3

Instituições de Ensino Superior (IES) e Critérios na Seleção de seus Pesquisadores Considerados neste Estudo

\begin{tabular}{|c|c|c|c|}
\hline $\begin{array}{l}\text { Nota } \\
\text { CAPES }\end{array}$ & Instituição de Ensino Superior (IES) & Cidade - UF & Critério de Seleção \\
\hline 7 & FGV/EAESP - Fundação Getulio Vargas SP & São Paulo - SP & 1. PPG com Linha de Pesquisa \\
\hline 7 & USP - Universidade de São Paulo & São Paulo - SP & 1. PPG com Linha de Pesquisa \\
\hline 6 & FGV/EBAPE - Fundação Getulio Vargas RJ & Rio de Janeiro - RJ & 4. Publicação JISTEM/RESI \\
\hline 5 & Universidade Federal do Rio Grande do Sul & Porto Alegre - RS & 1. PPG com Linha de Pesquisa \\
\hline 5 & PUC / PR & Curitiba - PR & $\begin{array}{l}\text { 2. Coordenação ou Liderança } \\
\text { de trilha }\end{array}$ \\
\hline 5 & PUC / RS & Porto Alegre - RS & $\begin{array}{l}\text { 2. Coordenação ou Liderança } \\
\text { de trilha }\end{array}$ \\
\hline 5 & UnB - Universidade de Brasília & Brasília - DF & 4. Publicação JISTEM/RESI \\
\hline 5 & UNINOVE - Universidade 9 de Julho & São Paulo - SP & $\begin{array}{l}\text { 2. Coordenação ou Liderança } \\
\text { de trilha }\end{array}$ \\
\hline 5 & $\begin{array}{l}\text { UNISINOS - Universidade do Vale do Rio dos } \\
\text { Sinos }\end{array}$ & São Leopoldo - RS & $\begin{array}{l}\text { 2. Coordenação ou Liderança } \\
\text { de trilha }\end{array}$ \\
\hline 5 & Universidade Federal da Paraíba & João Pessoa - PB & $\begin{array}{l}\text { 2. Coordenação ou Liderança } \\
\text { de trilha }\end{array}$ \\
\hline 5 & Universidade Federal de Lavras & Lavras - MG & $\begin{array}{l}\text { 3. Publicação } \\
\text { EnANPAD/EnADI }\end{array}$ \\
\hline 5 & USP - São Carlos & São Carlos - SP & 4. Publicação JISTEM/RESI \\
\hline 4 & IBMEC & Rio de Janeiro - RJ & 4. Publicação JISTEM/RESI \\
\hline 4 & Universidade Federal de Pernambuco & Recife - PE & $\begin{array}{l}\text { 2. Coordenação ou Liderança } \\
\text { de trilha }\end{array}$ \\
\hline 4 & Universidade Federal de Santa Catarina & Florianópolis - SC & $\begin{array}{l}\text { 3. Publicação } \\
\text { EnANPAD/EnADI }\end{array}$ \\
\hline 4 & $\begin{array}{l}\text { Universidade Federal do Estado do Rio de } \\
\text { Janeiro }\end{array}$ & Rio de Janeiro - RJ & $\begin{array}{l}\text { 2. Coordenação ou Liderança } \\
\text { de trilha }\end{array}$ \\
\hline 4 & USP - Ribeirão Preto & Ribeirão Preto - SP & $\begin{array}{l}\text { 3. Publicação } \\
\text { EnANPAD/EnADI }\end{array}$ \\
\hline 3 & Universidade de Santa Cruz do Sul & Santa Cruz - RS & $\begin{array}{l}\text { 2. Coordenação ou Liderança } \\
\text { de trilha }\end{array}$ \\
\hline 3 & Universidade do Estado do Rio de Janeiro & Rio de Janeiro - RJ & 3. Publicação EnANPAD/EnADI \\
\hline 3 & Universidade Federal Fluminense & Rio de Janeiro - RJ & 4. Publicação JISTEM/RESI \\
\hline
\end{tabular}

Nota. Fonte: Elaborada pelos autores.

RAC, Rio de Janeiro, v. 21, n. 6, art. 4, pp. 811-831 Novembro/Dezembro, 2017, www.anpad.org.br/rac (cc)E 
Na Tabela 3 pode-se observar a lista de instituições que mantêm pesquisadores considerados nesta pesquisa. Os 50 pesquisadores selecionados como os mais internacionalizados do campo de SI estão majoritariamente vinculados às instituições de ensino localizadas nas regiões Sudeste (66\%) e Sul (28\%) do País. A seguir apresentamos o perfil de atuação internacional dos pesquisadores brasileiros de SI com base nas dimensões Formação, Disseminação e Colaboração.

\section{Formação internacional}

Com relação à Formação no exterior dos 50 pesquisadores identificados, 37 participaram de algum curso no exterior ao longo da sua formação, sendo que 8 concluíram Doutorado completo no exterior (2 nos EUA, 2 na França, 2 em Portugal, 1 no Canadá e 1 no Reino Unido), outros 9 fizeram Doutorado Sanduíche (4 nos EUA, 2 na França, 2 no Reino Unido e 1 na Alemanha) e 19 fizeram Pósdoutorado. Observa-se também que o Pós-Doutoramento no exterior foi cursado predominantemente em instituições localizadas nos EUA (8 pesquisadores) e França (6 pesquisadores).

\section{Disseminação internacional}

A Figura 2 apresenta a evolução da publicação de artigos internacionais (periódicos e congressos) dos 50 pesquisadores brasileiros analisados neste estudo. A Disseminação internacional entre 2010 e 2015 cresceu $65 \%$ (de 64 para 106 artigos) e atingiu um total de 522 trabalhos publicados por estes pesquisadores, sendo 156 artigos publicados em periódicos e 366 artigos publicados em congressos.

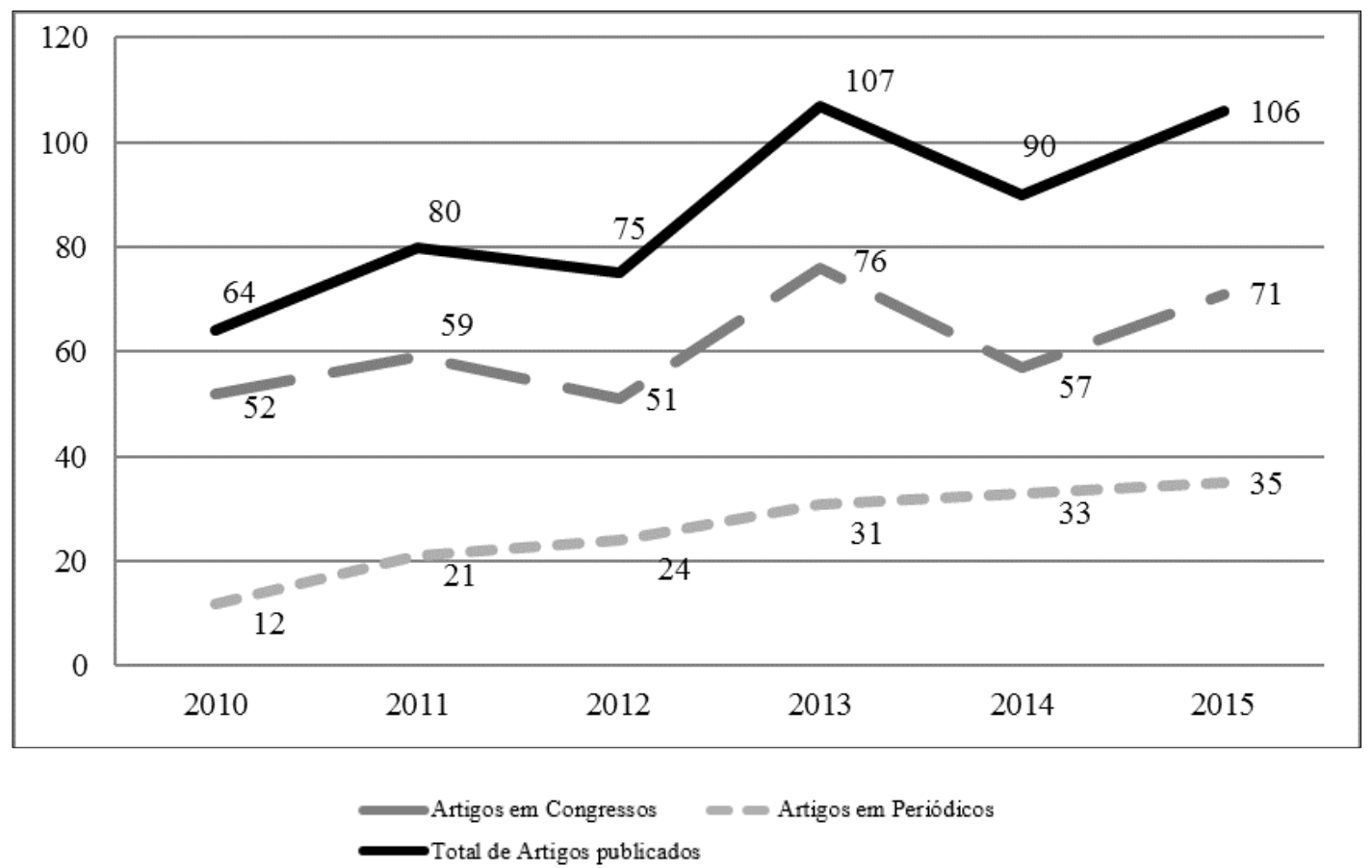

Figura 2. Evolução da Publicação de Artigos em Periódicos e Congressos Internacionais (Período de 2010 a 2015) dos 50 Pesquisadores Brasileiros do Campo de Estudos de si Analisados neste Estudo Fonte: Elaborada pelos autores.

Para os 156 artigos publicados em um total de 91 periódicos diferentes, foi observado que há uma concentração de pouco mais de $60 \%$ das publicações em apenas 34 periódicos. A Tabela 4 apresenta a lista de 13 periódicos internacionais que receberam ao menos 3 trabalhos de pesquisadores brasileiros no período de 2010 a 2015. Destaca-se a quantidade de artigos publicados (Qtd. de Artigos), o número ISSN e nome do periódico, além da classificação Qualis 2014 (CAPES, 2016b), Scimago Journal Rank 2015 (SJR, 2017) e Impact Factor - Thomson Reuters Journal Citation Reports (JCR, 2017) destes periódicos. 
Tabela 4

Lista dos Periódicos que Receberam ao Menos 3 Publicações de Pesquisadores Brasileiros (de 2010 a 2015)

\begin{tabular}{cllccc}
\hline $\begin{array}{c}\text { Qtd. } \\
\text { Artigos }\end{array}$ & ISSN & Nome do Periódico Internacional/Classificação & $\begin{array}{c}\text { Qualis } \\
\mathbf{2 0 1 4}\end{array}$ & $\begin{array}{c}\text { SJR } \\
\mathbf{2 0 1 5}\end{array}$ & $\begin{array}{c}\text { IF (JCR) } \\
\mathbf{2 0 1 5}\end{array}$ \\
\hline 11 & $0798-1015$ & Espacios & B1 & 0,156 & ND \\
\hline 6 & $0718-2724$ & Journal of Technology Management \& Innovation & B1 & 0,247 & ND \\
\hline 5 & $1062-7375$ & Journal of Global Information Management & A1 & 0,266 & 0,303 \\
\hline 5 & $1681-4835$ & $\begin{array}{l}\text { The Electronic Journal on Information Systems in } \\
\text { Developing Countries }\end{array}$ & B2 & 0,265 & ND \\
\hline 4 & $0747-5632$ & Computers in Human Behavior & A2 & 1,646 & 2,880 \\
\hline 4 & $1993-8233$ & African Journal of Business Management & B3 & ND & ND \\
\hline 4 & $1466-4437$ & Journal of Information Technology & ND & 1,474 & 1,695 \\
\hline 3 & $1548-0992$ & $\begin{array}{l}\text { Revista IEEE América Latina (IEEE Latin America } \\
\text { Transactions) }\end{array}$ & B2 & 0,249 & 0,436 \\
\hline 3 & $1567-4223$ & Electronic Commerce Research and Applications & ND & 1,582 & 2,139 \\
\hline 3 & $1550-1876$ & $\begin{array}{l}\text { International Journal of Information and } \\
\text { Communication Technology Education }\end{array}$ & B2 & 0,179 & ND \\
\hline 3 & $1865-0929$ & $\begin{array}{l}\text { Communications in Computer and Information } \\
\text { Science }\end{array}$ & B3 & 0,149 & ND \\
\hline 3 & $0740-624 X$ & Government Information Quarterly & A2 & 1,376 & 2,515 \\
\hline 3 & $1047-8310$ & $\begin{array}{l}\text { Journal of High Technology Management Research } \\
\text { A1 }\end{array}$ & A, & 0,342 & ND \\
\hline
\end{tabular}

Nota. ND = não disponível na respectiva classificação; Qualis 2014 = Qualis CAPES (Plataforma Sucupira) 2014; SJR 2015 = SCImago Journal Rank; IF (JCR) 2015 = Impact Factor (Thomson Reuters Journal Citation Reports). Fonte: Elaborada pelos autores.

Sobre o perfil desses periódicos, foi observado que, dentre os 121 que possuíam classificação Qualis 2014, 66\% estavam nos estratos B1, B2 e B3, 18\% em A2 e apenas 16\% classificados como no estrato A1. Para aqueles periódicos não identificados nas respectivas classificações, a tabela informa o rótulo $\mathrm{ND}$, ou seja, não disponível.

Já para os 366 artigos publicados em congressos internacionais (edições realizadas fora do Brasil), foi percebida a distribuição destes em 133 congressos diferentes, sendo que $60 \%$ dos trabalhos apresentados estão concentrados em apenas 20 congressos. A Tabela 5 apresenta essa lista de congressos internacionais onde foram apresentados pelo menos 4 trabalhos com autores brasileiros, no período analisado neste estudo (2010-2015). Os congressos internacionais eventualmente realizados no Brasil não foram considerados para esse levantamento. 
Tabela 5

Lista de Congressos que Receberam ao Menos 4 Publicações de Pesquisadores Brasileiros (de 2010 a 2015)

\begin{tabular}{|c|c|}
\hline Qtd Artigos & Nome do Congresso Internacional \\
\hline 54 & AMCIS - Americas Conference on Information Systems \\
\hline 23 & Conf-IRM - International Conference on Information Resources Management \\
\hline 15 & IFIP - e-Government Conference \\
\hline 14 & AOM - Academy of Management Annual Meeting \\
\hline 13 & HICSS - Hawaii International Conference on System Sciences \\
\hline 11 & ECIS - European Conference on Information Systems \\
\hline 10 & PICMET - Portland International Center for Management of Engineering and Technology \\
\hline 8 & ALTEC - Congresso Latino-Ibero-Americano de Gestão de Tecnologia \\
\hline 8 & ICEIS - International Conference on Enterprise Information Systems \\
\hline 8 & CAPSI - Conferência da Associação Portuguesa de Sistemas de Informação \\
\hline 8 & EGOS Colloquium \\
\hline 7 & ECKM - European Conference on Knowledge Management \\
\hline 6 & IAM - Iberoamerican Academy of Management \\
\hline 6 & IADIS - International Association for Development of the Information Society \\
\hline 5 & SIG GlobDev - Annual Workshop ICT in Global Development \\
\hline 5 & ICIS - International Conference on Information Systems \\
\hline 5 & IAMOT - International Management of Technology Annual Conference \\
\hline 5 & DG.O - Annual International Conference on Digital Government Research \\
\hline 5 & $\begin{array}{l}\text { IFIP WG } 9.4 \text { - International Conference on Social Implications of Computers in Developing } \\
\text { Countries }\end{array}$ \\
\hline 4 & GITMA - Annual Global Information Technology Management Association \\
\hline
\end{tabular}

Nota. Fonte: Elaborada pelos autores.

\section{Colaboração internacional}

$\mathrm{Na}$ dimensão Colaboração internacional, foram consideradas, dentre os pesquisadores selecionados, as atuações em projetos de pesquisa internacionais, em comitês científicos de congressos ou em comitês editoriais de periódicos, participação em associações, e atuação eventual na revisão de artigos para congressos e periódicos, todas no âmbito internacional.

Foram observadas atuações dos pesquisadores selecionados em projetos de pesquisa realizados com parceria internacional, de forma mais concentrada em 5 diferentes países (Alemanha, EUA, Canadá, Inglaterra e Holanda), como descrito pela Tabela 6. 
Tabela 6

Lista de Países e suas Instituições com Parcerias em Projetos de Pesquisa Internacional (2010 a 2015)

\begin{tabular}{cll}
\hline $\begin{array}{c}\text { Qtd de } \\
\text { Pesquisadores }\end{array}$ & País de Origem & Nomes das Instituições de Ensino do Exterior \\
\hline $\mathbf{8}$ & Alemanha & $\begin{array}{l}\text { University of Münster; Universität Lüneburg (Leuphana); University of } \\
\text { Heidelberg; Universität Münster }\end{array}$ \\
\hline $\mathbf{7}$ & EUA & $\begin{array}{l}\text { University of North Carolina at Greensboro; University of Texas at El } \\
\text { Paso; Canisius College; University of North Carolina Wilmington; } \\
\text { Florida Atlantic University; College of Charleston; University of Texas } \\
\text { at Austin; University of California }\end{array}$ \\
\hline $\mathbf{2}$ & Canadá & International Development Research Center; Carleton University \\
\hline $\mathbf{2}$ & Inglaterra & London School of Economics and Political Science; University of Leeds \\
\hline $\mathbf{2}$ & Inglaterra/ Holanda & University of Stirling / Utrecht University School of Governance \\
\hline
\end{tabular}

Nota. Fonte: Elaborada pelos autores.

A Tabela 6 descreve, além da quantidade de pesquisadores, os países e as principais instituições internacionais parceiras em projetos de pesquisa com brasileiros, entre 2010 e 2015 . Ficou evidenciado que a rede de Colaboração internacional deste grupo de pesquisadores se concentra prioritariamente em parcerias com pares nas universidades da Alemanha e dos EUA. Como destaque dos demais quesitos avaliados nesta dimensão, a participação destes 50 pesquisadores como revisores de artigos em periódicos internacionais ficou mais evidenciada em dois países: Inglaterra e EUA.

\section{Discussão dos Resultados}

Este artigo apresentou os resultados de um estudo que pretende decifrar as estratégias de inserção internacional dos pesquisadores brasileiros do campo de ADI. Como resultados da investigação feita, observa-se que a Formação no exterior tem papel muito relevante na articulação internacional dos pesquisadores, embora, surpreendentemente, a participação em programas de post-doc e de bolsa sanduíche pareça contribuir mais do que concluir o doutorado completo em outro país. A pressão por publicação tem surtido efeito nesta comunidade de pesquisadores, que têm ampliado significativamente a Disseminação de suas pesquisas no exterior. Embora poucos ainda publiquem em periódicos de primeira linha do campo, a participação em congressos mais relevantes começa a se fazer mais constante. A presença em redes de colaboração internacional é ainda restrita, mas parece ter forte relação com a presença dos pesquisadores em congressos mais importantes.

A partir da combinação das três dimensões estudadas - Formação, Disseminação e Colaboração, é possível conhecer o perfil da atuação internacional dos pesquisadores de ADI, como uma primeira etapa para se avaliar a presença brasileira no campo de estudos de Information Systems e o potencial para ampliar sua relevância. A partir dessa análise, pode-se avaliar novos mecanismos de incentivo, políticas de acesso e priorização e outras medidas que contribuam para o crescimento da contribuição da pesquisa brasileira no cenário internacional.

Quanto à Formação, surpreende que uma parcela significativa (13 em 50) dos principais pesquisadores da área não tenham tido nenhuma experiência internacional. Esse número pode conter um viés de informação desatualizada no Currículo Lattes, mas dada a sua importância, esse é um ponto que deve ser acompanhado de perto. A Bolsa sanduíche (9 em 50) ainda não parece também ter sido um canal relevante para promover a internacionalização dos pesquisadores do campo. Por outro lado, $38 \%$ desses pesquisadores (19 em 50) tiveram, em contrapartida, algum tipo de experiência internacional 
após o doutorado (post-doc), o que é mais significativo. E neste caso já aparecem brasileiros em instituições que estão na primeira linha em SI.

Sobre a Disseminação, os pesquisadores brasileiros parecem melhor sintonizados em relação à participação nos principais congressos internacionais, particularmente se for considerado o ranking dos cinco congressos nos quais foram identificados maior número de pesquisadores do país, todos muito relevantes para o campo. Por outro lado, há que se constatar que os pesquisadores brasileiros ainda estão longe de apresentar uma boa posição quando se trata de publicação em periódicos relevantes. Dois terços dos periódicos internacionais alvo das submissões dos pesquisadores brasileiros estão nos estratos B1, B2 e B3.

Ainda que se louve o fato de serem identificados, nessa lista de 50 pesquisadores, 7 artigos publicados em periódicos da senior basket, cabe a reflexão para futura investigação se é a cultura local que dirige o foco dos trabalhos de Disseminação em congressos, ou se essa atitude é fruto da falta de maior empenho para direcionar os estudos para os top journals do campo, ou, ainda, se submissões para esses periódicos foram feitas, mas sem sucesso. As estratégias de Disseminação dos pesquisadores brasileiros têm a ver com os periódicos e congressos regionais, como as revistas African Journal of Business Management e a venezuelana Espacios (Tabela 4), e os congressos ALTEC e o português CAPSI (Tabela 5). Essa busca de integração regional é também louvável e merece ser investigada com mais profundidade.

Da Colaboração internacional, observa-se que 8 pesquisadores desse grupo analisado possuem parcerias com pares de instituições alemãs e 7 com instituições dos EUA (Tabela 6). Se parece lógica a intensa parceria com os norte-americanos, surpreende o nível de parceria com os colegas alemães. É o caso de aprofundar os motivos para essa situação, que talvez possa ser explicada por parcerias entre os próprios organismos nacionais de financiamento, como CNPq e FAPESP, mas também pode ter origem no fato de este país europeu estar mais orientado a financiar pesquisas com parceria internacional no campo. De qualquer forma, essas parcerias são fortemente orientadas pela disponibilização de recursos específicos.

Quanto à participação em comitês de congressos e periódicos além das associações científicas, que não envolve empenho de recursos significativos, nota-se que os pesquisadores brasileiros são muito mais ativos, demonstrando uma clara orientação para participar em redes internacionais. Também neste caso uma futura análise quantitativa que descubra relações entre os dados se faz necessária para o aprofundamento sobre o comportamento dos pesquisadores brasileiros em redes internacionais. No caso da dimensão Colaboração, nota-se igualmente a participação em redes regionais, tanto nos projetos quanto na participação em comitês e associações. Uma possível alternativa de participação em grupos de pesquisa internacional seria buscar o ambiente mainstream da pesquisa - portanto, com foco nos EUA e Europa, aumentando as possibilidades de publicação em periódicos de ponta -, não se desprezando, entretanto, as oportunidades de articulação regional com colegas de países fora dos grandes centros.

\section{Considerações Finais e Limitações deste Estudo}

Pesquisas bibliométricas para estudar como a ciência de SI têm evoluído, seu processo de progresso na construção do conhecimento do campo, observado seja pelas ações de internacionalização tomadas por seus pesquisadores e suas instituições de ensino, seja pela publicação de artigos, tanto em periódicos quanto em congressos internacionais, tornando-se valiosos guias para sinalizar o estado atual e futuro do próprio campo. Também foi observado, pela literatura acadêmica (Straub, 2006), que autores de estudos bibliométricos podem chegar a conclusões radicalmente diferentes quando optam por critério de incluir ou remover determinado periódico de uma lista definida para o estudo, alterando significantemente seus achados. Neste aspecto, por esta pesquisa ser baseada em dados e informações 
provenientes principalmente dos Currículos Lattes dos pesquisadores, como limitação do estudo, pode haver um viés de informação desatualizada, apesar dos cuidados metodológicos adotados.

Os resultados do estudo apresentado neste artigo são bastante reveladores sobre as estratégias utilizadas pelos pesquisadores brasileiros do campo de estudos de ADI que buscam inserção internacional e também sobre as possíveis relações entre as três dimensões estudadas, como uma interessante contribuição para entender a dinâmica de internacionalização deste campo no País. Como contribuição teórica, acredita-se que o modelo de análise utilizado e o estudo de suas relações possam ser explorados para se entender o processo de internacionalização, não apenas de pesquisadores do campo específico de SI, mas também de outros campos de estudo ou mesmo de outras áreas de conhecimento além da Administração.

Os números apresentados, entretanto, revelam apenas o passado, pois a coleta de dados foi feita em bases secundárias. Há que se investigar como esses mesmos pesquisadores estão planejando hoje a sua inserção internacional, e não apenas o que já foi feito. Para tanto, esses pesquisadores podem ser abordados diretamente e confrontados com esses dados com o objetivo de identificar a estratégia que está sendo desenhada para os próximos anos e que não pode ser capturada completamente apenas com dados do passado. Outra limitação da pesquisa está relacionada ao fato de o modelo desenvolvido abordar apenas a atuação do pesquisador como indivíduo e não considerar as contribuições para a sua internacionalização a partir de estratégias institucionais e do contexto no qual o pesquisador está inserido. Esta limitação também restringe qualquer análise que possa ser feita com o objetivo de apontar recomendações de políticas públicas para incentivar a internacionalização. Este é um aspecto que deve ser melhor explorado em futuros estudos, que podem incluir surveys com pesquisadores, análise de políticas institucionais e entrevistas com pesquisadores que tenham atingido um patamar mais alto de internacionalização.

\section{Referências}

Associação Nacional de Pós-Graduação e Pesquisa em Administração. (n.d.). Encontro Nacional da Associação Nacional de Pós-Graduação e Pesquisa em Administração. Recuperado em 20 de janeiro, 2017, de http://www.anpad.org.br/ anpad/eventos.php?cod_evento=1\#

Association for Information Systems. (n.d.b). Senior scholars' basket of journals. Retrieved January 20, 2017, from http://aisnet.org/?page=SeniorScholarBasket

Association for Information Systems. (n.d.a). The AIS faculty directory. Retrieved January 20, 2017, from https://aisnet.org/?FacultyDirectory

Benbasat, I., \& Zmud, R. W. (2003). The identity crisis within the IS discipline: Defining and communicating the discipline's core properties. MIS Quarterly, 27(2), 183-194.

Bernroider, E., Pilkington, A., \& Córdoba, J. R. (2013). Research in information systems: A study of diversity and inter-disciplinary discourse in the AIS basket journals between 1995 and 2011. Journal of Information Technology, 28(1), 74-89. http://dx.doi.org/10.1057/jit.2013.5

Bichler, M., Heinzl, A., \& Winter, R. (2015). Practice impact of IS research. Business \& Information Systems Engineering, 57(2), 87-89. http://dx.doi.org/10.1007/s12599-015-0369-1

Bryant, A., Black, A., Land, F., \& Porra, J. (2013). Information systems history: What is history? What is IS history? What IS history?... and why even bother with history? Journal of Information Technology, 28(1), 1-17. http://dx.doi.org/10.1057/jit.2013.3 
Chan, H. C., Guness, V., \& Kim, H. W. (2015). A method for identifying journals in a discipline: An application to information systems. Information \& Management, 52(2), 239-246. http://dx.doi.org/10.1016/j.im.2014.11.003

Chua, C., Cao, L., Cousins, K., \& Straub, D. (2002). Measuring researcher-production in information systems. Journal of the Association for Information Systems, 3(1), 145-215.

Clark, J., Au, Y., Walz, D., \& Warren, J. (2011). Assessing researcher publication productivity in the leading information systems journals: A 2005-2009 Update. Communications of the Association for Information Systems, 29(26), 459-502.

Clarke, R. (2008). An exploratory study of information systems researcher impact. Communications of the Association for Information Systems, 22(1), 1-32.

Conselho Nacional de Desenvolvimento Científico e Tecnológico. (n.d.). Currículo Lattes. Recuperado em 20 janeiro, 2017, de http://buscatextual.cnpq.br/buscatextual/busca.do?metodo=apresentar

Coordenação de Aperfeiçoamento de Pessoal de Nível Superior. (2016a). Cursos recomendados e reconhecidos. Recuperado em 20 janeiro, 2017, de https://sucupira.capes.gov.br/sucupira/public/consultas/coleta/programa/quantitativos/quantitati voConceito.jsf

Coordenação de Aperfeiçoamento de Pessoal de Nível Superior. (2016b). Plataforma Sucupira - Qualis periódicos. Recuperado em 20 janeiro, 2017, de https://sucupira.capes.gov.br/sucupira/public/consultas/coleta/veiculoPublicacaoQualis/listaCon sultaGeralPeriodicos.jsf

Coordenação de Aperfeiçoamento de Pessoal de Nível Superior. (2017). Administração pública e de empresas, ciências contábeis e turismo. Recuperado em 20 janeiro, 2017, de https://www.capes.gov.br/component/content/article/44-avaliacao/4649-administracao-cienciascontabeis-e-turismo

Dennis, A., Valacich, J., Fuller. M., \& Schneider, C. (2006). Research standards for promotion and tenure in information systems. MIS Quarterly, 30(1), 1-12. http://dx.doi.org/10.2307/25148714

Dias, T. M. R., Moita, G. F., \& Dias, P. M. (2016). Adoção da plataforma lattes como fonte de dados para caracterização de redes científicas. Encontros Bibli: Revista Eletrônica de Biblioteconomia e Ciência da Informação, 21(47), 16-26. Recuperado de https://periodicos.ufsc.br/index.php/eb/article/download/1518-2924.2016v21n47p16/32330. http://dx.doi.org/10.5007/1518-2924.2016v21n47p16

Diniz, E. H., Favaretto, J. E. R., Oliveira, H. P. G., \& Brólio, D. V. R. (2016, setembro). Inserção internacional do campo de administração da informação (ADI): Análise da formação, publicação e participação em redes de pesquisa. Anais do Encontro Nacional da Associação Nacional de Pós-Graduação e Pesquisa em Administração, Costa do Sauípe, BA, Brasil, 40.

Eisend, M., \& Schmidt, S. (2014). The influence of knowledge-based resources and business scholars'internationalization strategies on research performance. Research Policy, 43(1), 48-59. http://dx.doi.org/10.1016/j.respol.2013.07.011

Freitas, H., Becker, J., Martens, C. D. P., \& Marcolin, C. (2014). Sistemas de informação - Temas de pesquisa acadêmica no Brasil entre 1994 e 2013. Revista Eletrônica de Sistemas de Informação, 13(3). http://dx.doi.org/10.5329/RESI.2014.1303001

Gallivan, M., \& Ahuja, M. (2015). Co-authorship, homophily, and scholarly influence in information systems research. Journal of the Association for Information Systems, 16(12), 980-1015. 
Gannon, B. (2013). Outsiders: An exploratory history of IS in corporations. Journal of Information Technology, 28(1), 50-62. http://dx.doi.org/10.1057/jit.2013.2

Google Scholar. (n.d.). Google Acadêmico - Perfis. Recuperado em 20 de janeiro, 2017, de https://scholar.google.com.br/citations?view_op=search_authors\&hl=ptBR\&mauthors=label:sistemas_de_informa\%C3\%A7\%C3\%A3o

Graeml, A., \& Macadar, M. (2010). Análise de citações utilizadas em ADI: 10 anos de anais digitais do EnANPAD (1997-2006). Revista de Administração Contemporânea, 14(1), 122-148. Recuperado de http://www.scielo.br/pdf/rac/v14n1/08.pdf. http://dx.doi.org/10.1590/S141565552010000100008

Graeml, A., Macadar, M., Guarido, E., \& Rossoni, L. (2010). Redes sociais e intelectuais em administração da informação: Uma análise cientométrica do período 1997-2006. Informação \& Sociedade: Estudos, 20(1), 95-110.

Grover, V., \& Lyytinen, K. (2015). New state of play in information systems research: The push to the edges. MIS Quarterly, 39(2), 271-296. http://dx.doi.org/10.25300/MISQ/2015/39.2.01

Heinzl, A., Winter, R., \& Bichler, M. (2015). Internationalization of information systems research and teaching. Business \& Information Systems Engineering, 57(4), 225-228. http://dx.doi.org/10.1007/s12599-015-0388-y

Hirschheim, R., \& Klein, H. K. (2012). A glorious and not-so-short history of the information systems field. Journal of the Association for Information Systems, 13(4), 188-235.

Hirschheim, R., Saunders, C., \& Straub, D. (2012). Historical interpretations of the IS discipline: An introduction to the special issue. Journal of the Association for Information System, 13(4), i-viii.

Hoppen, N., \& Meirelles, F. S. (2005). Sistemas de informação: Um panorama da pesquisa científica entre 1990 e 2003. Revista de Administração de Empresas, 45(1), 24-35. http://dx.doi.org/10.1590/S0034-75902005000100004

Ives, B., Valacich, J., Watson, R. T., \& Zmud, R. (2002). What every business student needs to know about information systems. Communications of the Association for Information Systems, 9(1), 467-477.

Journal Citation Reports. (2017). InCites Journal Citation Reports - Thomson Reuters (Clarivate Analytics). Retrieved January 20, 2017, from https://jcr.incites.thomsonreuters.com

Journal of Information Systems and Technology Management. (n.d.). Search. Retrieved January 20, 2017, from http://www.jistem.fea.usp.br/index.php/jistem/search/search

Katz, J. S., \& Martin, B. R. (1997). What is research collaboration? Research Policy, 26(1), 1-18. http://dx.doi.org/10.1016/S0048-7333(96)00917-1

King, J. L., \& Lyytinen, K. (2006). (Eds). Information systems: The state of the field. Chichester: John Wiley \& Sons Ltd.

Lane, J. I. (2010). Let's make science metrics more scientific. Nature, 464(7288), 488-489. http://dx.doi.org/10.1038/464488a

Lin, A., \& Gregor, S. (2009). Publication productivity in information systems 2003-2007: A Focus on the 'Basket of Six' and the Pacific Asia Region. Pacific Asia Journal of the Association for Information Systems, 1(1), 1-16. 
Macadar, M. A., \& Graeml, A. R. (2010). Refletindo sobre a área de ADI: O que pensam os pesquisadores da área? Revista Eletrônica de Administração, 16(2), 348-372. Recuperado de http://seer.ufrgs.br/read/article/viewFile/38852/25024

Meneghini, R., Packer, A. L., \& Nassi-Calo, L. (2008). Articles by Latin American authors in prestigious journals have fewer citations. PLoS One, 3(11), e3804. http://dx.doi.org/10.1371/journal.pone.0003804

Mumford, E. (2006). The story of socio-technical design: Reflections on its successes, failures and potential. Information Systems Journal, 16(4), 317-342. http://dx.doi.org/10.1111/j.13652575.2006.00221.x

Orlikowski, W. J., \& Iacono, C. S. (2001). Research commentary: Desperately seeking the "IT" in IT research - A call to theorizing the IT artifact. Information Systems Research, 12(2), 121-134. http://dx.doi.org/10.1287/isre.12.2.121.9700

Perlin, M. S., Santos, A. A. P., Imasato, T., Borenstein, D., \& Silva, S. da (2017). The Brazilian scientific output published in journals: A study based on a large CV database. Journal of Informetrics, 11(1), 18-31. http://dx.doi.org/10.1016/j.joi.2016.10.008

Pozzebon, M., Diniz, E., \& Reinhard, N. (2011). Creating a Brazilian school in international information systems research: Opportunities and challenges. Revista de Administração de Empresas, 51(1), 10-14. http://dx.doi.org/10.1590/S0034-75902011000100002

Pritchett, B. (2016, January 14). AIS welcomes Brazil chapter. Association for Information Systems, Atlanta. Retrieved January 20, 2017, from http://aisnet.org/news/269400/ais-welcomes-BrazilChapter.htm

Revista Eletrônica de Sistemas de Informação. (n.d.). Pesquisa. Recuperado em 20 janeiro, 2017, de http://www.periodicosibepes.org.br/index.php/reinfo/search/

Rodrigues, J., Filho, \& Ludmer, G. (2005). Sistema de informação: Que ciência é essa? Revista de Gestão da Tecnologia e Sistemas de Informação, 2(2), 151-166. http://dx.doi.org/10.4301/S180717752005000200004

Rossoni, L., \& Hocayen-da-Silva, A. J. (2009). Administração da informação: A produção científica brasileira entre 2001 e 2006. Revista Eletrônica de Administração, 15(2), 1-25. Recuperado de http://seer.ufrgs.br/index.php/read/article/download/39071/25055

Saunders, C. (2006). Editor's comments: What does it take for a journal to be global? MIS Quarterly, 30(3), iii-vi. http://dx.doi.org/10.2307/25148739

Scimago Journal \& Country Rank. (2017). Retrieved January 20, 2017, from http://www.scimagojr.com/journalsearch.php

Sidorova, A., Evangelopoulos, N., Valacich, J. S., \& Ramakrishnan, T. (2008). Uncovering the intellectual core of the information systems discipline. MIS Quarterly, 32(3), 467-482.

Stein, M.-K., Galliers, R., \& Whitley, E. (2016). Twenty years of the European information systems academy at ECIS: Emergent trends and research topics. European Journal of Information Systems, 25(1), 1-15. http://dx.doi.org/10.1057/ejis.2014.25

Straub, D. (2006). The value of scientometric studies: An introduction to a debate on IS as a reference discipline. Journal of the Association for Information Systems, 7(5), 241-245.

Straub, D. (2012). Editor's comments: Does MIS have native theories? MIS Quarterly, 36(2), iii-xii. 
Wade, M., Biehl, M., \& Kim, H. (2006). Information systems is not a reference discipline (and what we can do about it). Journal of the Association for Information Systems, 7(5), 247-269.

Weber, R. (2012). Evaluating and developing theories in the information systems discipline. Journal of the Association for Information Systems, 13(1), 1-30.

Willcocks, L. P., Whitley, E. A., \& Avgerou, C. (2008). The ranking of top IS journals: A perspective from London School of Economics. European Journal of Information Systems, 17(2), 163-168. http://dx.doi.org/10.1057/ejis.2008.9

Xu, J., Chau, M., \& Tan, B. (2014). The development of social capital in the collaboration network of information systems scholars. Journal of the Association for Information Systems, 15(12), 835859.

Zhang, P. (2015). The IS history initiative: Looking forward by looking back. Communications of the Association for Information Systems, 36(24), 477-514.

Zmud, B. (1998). Editor's comments: "Pure" theory manuscripts. MIS Quarterly, 22(2), xxix-xxxii.

\section{Dados dos Autores}

Eduardo H. Diniz

Rua Itapeva, 474, 01313-902, São Paulo, SP, Brasil. E-mail: eduardo.diniz@fgv.br

José Eduardo Ricciardi Favaretto

Rua Itapeva, 474, 01313-902, São Paulo, SP, Brasil. E-mail: jose@favaretto.net

Henrique Pontes Gonçalves de Oliveira

Rua Itapeva, 474, 01313-902, São Paulo, SP, Brasil. E-mail: henrique.pontes@uol.com.br

Debora Richter Brólio

Rua Itapeva, 474, 01313-902, São Paulo, SP, Brasil. E-mail: debora.richter@gmail.com 